\title{
Phase-plate Cryo-electron Tomography Facilitates the Identification of Influenza Virus Condensed Core Structures
}

\author{
John Gallagher ${ }^{1}$, Neetu Gulati ${ }^{2}$ and Audray Harris ${ }^{2}$ \\ ${ }^{1}$ NIH / NIAID, Bethesda, Maryland, United States, ${ }^{2}$ NIH, Bethesda, Maryland, United States
}

Influenza effects millions of people globally each year. Previous studies of influenza internal structure used cryo-electron microscopy with primarily film and charged-coupled device (CCD) cameras as detectors indicated a pleiomorphic virus structure, with multiple structural layers [1-5]. Influenza virus structure is conserved among influenza A (e.g. widely circulating H1 and H3) and influenza B viruses. The virion architecture consists of viral glycoproteins hemagglutinin (HA), neuraminidase (NA) and M2 embedded in the viral membrane. Underneath the viral membrane is a matrix layer formed from the M1 matrix protein. Inside the virus are genomic ribonucleoprotein complexes (RNPs) that from packaged filaments constructed from viral RNA and the nucleoprotein (NP) [2, 4-8]. However, rare internal influenza solenoid structures termed a condensed core was identified by cryo-electron tomography [2]. Later low-pH studies of filamentous influenza viruses confirmed an internal condensed core structure [1]. The implications of this internal condensed core structure for influenza virus assembly and entry has been unknown. One reason is that the observation of the internal influenza condensed core structure is rare, while the majority of virions have unadorned filamentous genomic RNPs inside. The lack of additional structural information on how influenza internal proteins may condense to form a solenoid-like condensed core structure during assembly or uncoating hinders studies to further investigate the relationship of infectivity and internal structure reorganization. Here, we tested the ability of traditional 2D cryo-EM images collected via CCD versus cryo tomography using a direct detector with a Volta phase plate to more readily identify virons with an internal condensed core structures within influenza virus.

H1N1 2009 influenza A viruses were purified from infected Madin-Darby Canine Kidney (MDCK) cells (Figure 1). X-31 H3N2 influenza A viruses were purified from infected embryonated chicken eggs (Figure 2). For cryo-electron microcopy, 200 mesh R $2 / 2$ Quantifoil copper grids were glow discharged and then $3.5 \mu \mathrm{l}$ of unstained virus were applied. Cryo-electron tomography samples were mixed with $10 \mathrm{~nm}$ gold particles for use as fiducials. Sample grids were plunge-frozen with a Vitrobot Mark IV plunger (Thermo Fisher Scientific). For H1N1 influenza virus samples cryo-EM data was collected under cryo conditions at $300 \mathrm{kV}$ on a Titan Krios electron microscope (Thermo Fisher Scientific) using EPU software on a $4 \mathrm{k} \times$ 4k Gatan Ultrascan charge-coupled-device (CCD) with a 1.9 angstrom pixel size (nominal magnification $47,000 \mathrm{x})$. The electron dose ranged from $\sim 10$ to $20 \mathrm{e}-$-square angstrom. For H3N2 influenza virus tomography, tilt series collected with VPP on Falcon II (Thermo Fisher Scientific) was acquired at $2.9 \AA$ pixel size (nominal magnification 29,000x) with a $-2.5 \mu \mathrm{m}$ defocus. All single-axis tilt series data were collected over $-/+60$ degrees ( 2 degrees tilt increments) using Tomography 4.0 software. Datasets (i.e. tilt series) were collected under low-dose conditions with a cumulative electron dose of 100 electrons/square angstrom. Gold particles were used as fiducial markers to mutually align images with the tilt series and the 3D volumes (tomograms) were reconstructed as implemented the IMOD software package [9].

We found that influenza internal condensed cores could be observed in CCD data. However, the vast majority ( $99 \%$ ) of the influenza viruses had non-structured fuzzy density inside when observing 2D cryoEM images (Figure 1a-1c). Glycoprotein spikes could be observed on the surfaces of virus particles (Figure 1a-1c, black arrows). However, only about 1\% of particles had observable condensed cores inside (Figure 1d-1e). Glycoproteins still decorated the membrane in virions containing condensed cores. 
Condensed cores appeared as barrel-shaped structures inside the virions (Figure 1d-1f, white arrows). Interestingly, some particles had one, two, or three observable condensed cores. In addition, some particles displayed lesser glycoprotein spikes on their surfaces (Figure 1f). To test whether viruses grown in eggs and imaged with higher contrast could reveal more internal condensed cores, we carried out cryo-electron tomography on egg-grown $\mathrm{X}-31 \mathrm{H} 3 \mathrm{~N} 2$ virus using a phase plate. The contrast was increased with the phase plate, but the majority of the viruses had internal RNPs (Figure 2a, white arrows) and not condensed cores. In some viruses internal condensed cores where observed (Figure 2a, 2b, plus signs). Interestingly, some structural striations could be observed on a few of the internal condensed cores (e.g. Figure 2c). This suggests an ordered arrangement of structural constituents. Further studies on other viral strains coupled with automated data collection and particle selection with phase plate data could be useful in studying what role the internal condensed core of influenza plays in virus structure and function [10].

Cryo-EM of H1N1 influenza viruses

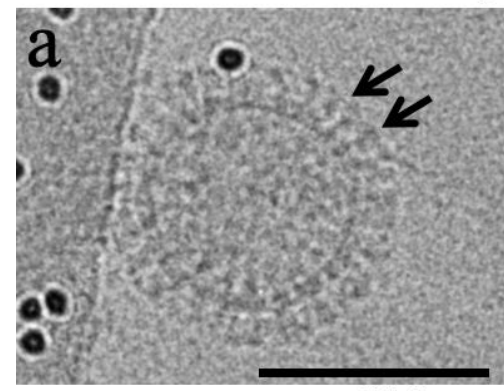

$100 \mathrm{~nm}$

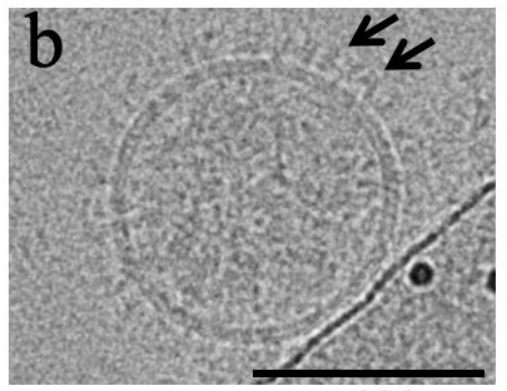

$100 \mathrm{~nm}$

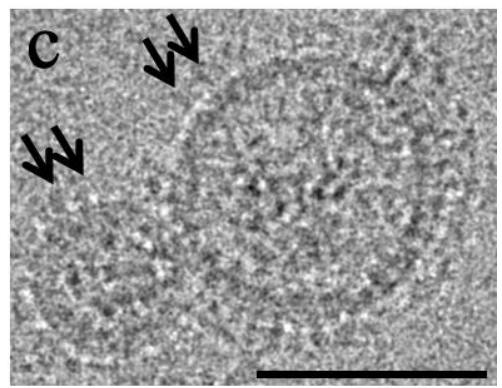

$100 \mathrm{~nm}$

\section{Cryo-EM of H1N1 influenza viruses (with condensed internal cores)}

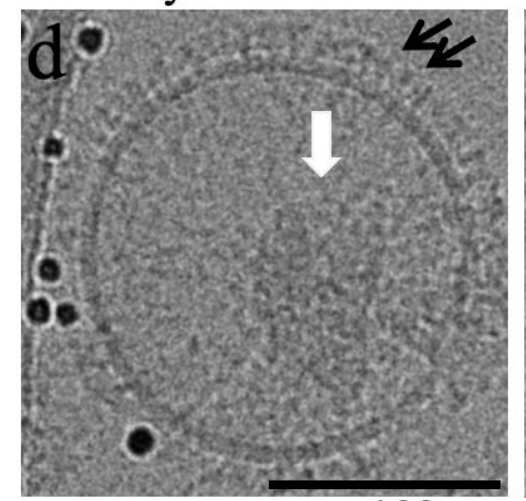

$100 \mathrm{~nm}$

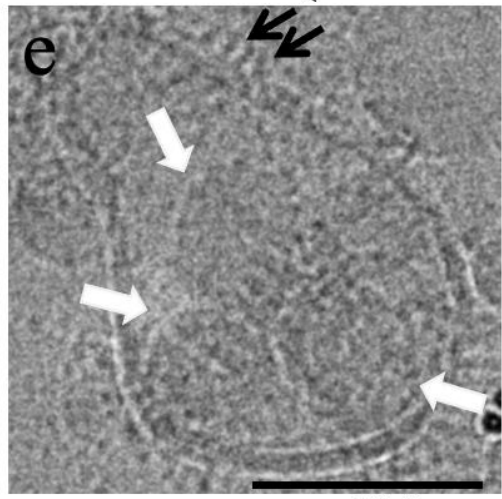

$100 \mathrm{~nm}$

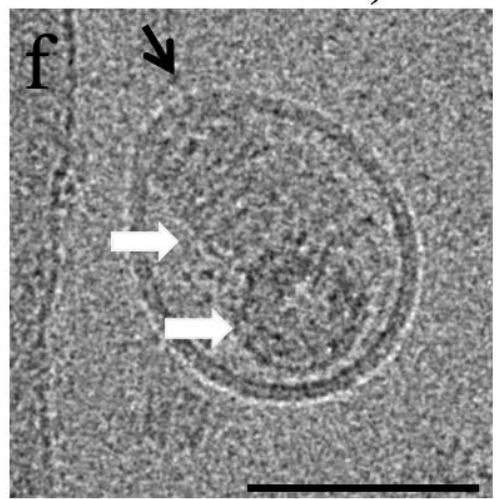

$100 \mathrm{~nm}$

Figure 1. Cryo-electron microscopy of influenza virus particles using a charged-coupled device (CCD) camera. $(\mathrm{a}, \mathrm{b}, \mathrm{c})$ Virus particles without observable internal cores. $(\mathrm{d}, \mathrm{e}, \mathrm{f})$ Virus particles with observable internal core structures. Black arrows denote glycoprotein spikes on the surfaces of the particles. White arrows denote internal core structures inside virus particles. Dark dots are nanogold particles used as fiducial markers in tomography experiments. The virus is 2009 H1N1 pandemic virus (A/California/07/2009 (H1N1)) purified from MDCK cells. Scale bars, $100 \mathrm{~nm}$. 


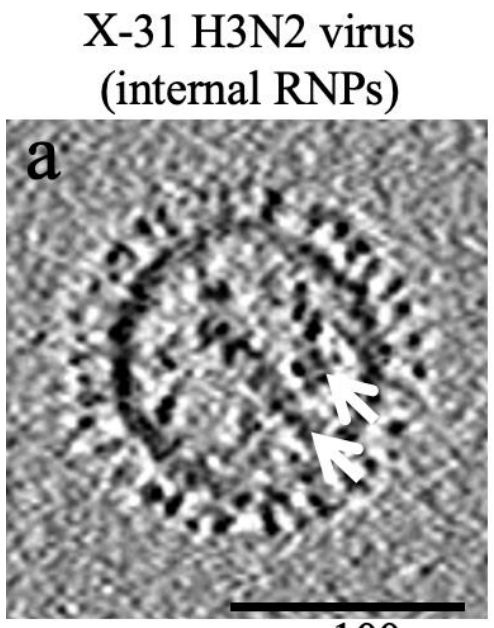

$100 \mathrm{~nm}$

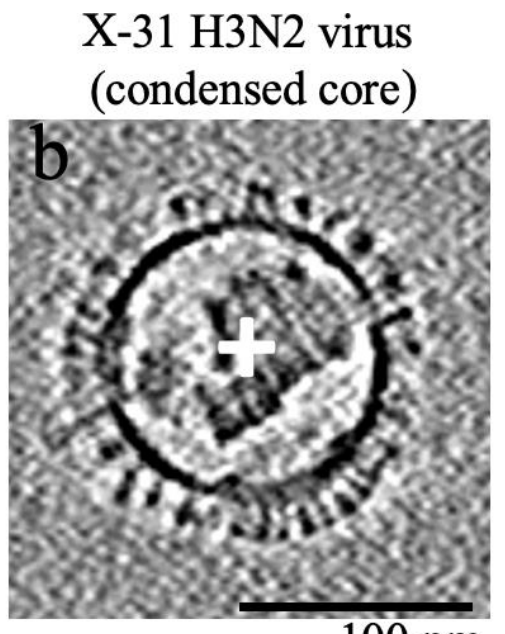

$100 \mathrm{~nm}$
$\mathrm{X}-31 \mathrm{H} 3 \mathrm{~N} 2$ virus (condensed core)

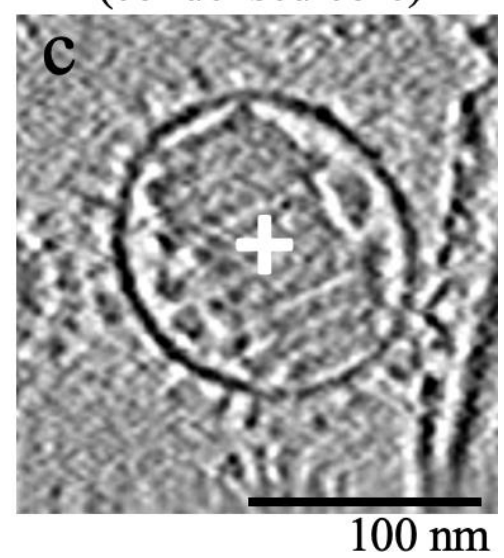

Figure 2. Cryo-electron tomography of influenza virus particles imaged using a Volta Phase-Plate (VPP) and direct electron detector. (a) A near central slice through 3D volume (tomogram) of a virus particle with internal genomic RNP filaments. Examples are denoted by white arrows. (b) A near central slice through a 3D volume (tomogram) of a virus particle with an internal core denoted by an white plus sign. (c) A near central slice through a 3D volume (tomogram) of a virus particle with an internal core with observable diagonal striations of the core structure. The core is denoted by a white plus sign. The virus is X-31 (H3N2) influenza virus purified from embryonated chicken eggs). Scale bars, $100 \mathrm{~nm}$.

\section{References}

[1] LJ Calder et al., Proc Natl Acad Sci U S A 107 (2010), p. 10685.

[2] A Harris et al., Proc Natl Acad Sci U S A 103 (2006), p. 19123.

[3] AK Harris et al., Proc Natl Acad Sci U S A 110 (2013), p. 4592.

[4] G Katz et al., PLoS One 9 (2014), p. e88288.

[5] S Vijayakrishnan et al., PLoS Pathog 9 (2013), p. e1003413.

[6] JR Gallagher et al., J Struct Biol, 10.1016/j.jsb.2016.12.007(2016), p.

[7] NM Gulati et al., Microscopy and microanalysis : the official journal of Microscopy Society of America, Microbeam Analysis Society, Microscopical Society of Canada 25 (2019), p. 1302.

[8] T Noda et al., Nat Commun 9 (2018), p. 54.

[9] JR Kremer, DN Mastronarde and JR McIntosh, J Struct Biol 116 (1996), p. 71.

[10] The authors acknowledge support from the Intramural Research Program of NIAID/NIH and V. Nair and E. Fischer at the NIAID Research Technologies Branch for aid in cryo-EM data collection. 